\title{
SARS-CoV-2-related Multisystem Inflammatory Syndrome in Adults
}

\author{
António Martins ${ }^{1,2}$, Sílvia Policarpo ${ }^{1,2}$, André Silva-Pinto ${ }^{1,2,3}$, Ana Sofia Santos ${ }^{1,2}$, \\ Paulo Figueiredo ${ }^{1,2}$, António Sarmento ${ }^{1,2,3}$, Lurdes Santos ${ }^{1,2,3}$ \\ ${ }^{1}$ Infectious Diseases Intensive Care Unit, Infectious Diseases Department, Centro Hospitalar Universitário de São João, Porto, Portugal \\ ${ }^{2}$ Department of Medicine, Faculdade de Medicina da Universidade do Porto, Porto, Portugal \\ ${ }^{3}$ Nephrology and Infectious Diseases R\&D, i3S - Instituto de Investigação e Inovação em Saúde da Universidade do Porto, Porto, Portugal
}

Received: 23/10/2021

Accepted: 08/11/2021

Published: 26/11/2021

\begin{abstract}
How to cite this article: Martins A, Policarpo S, Silva-Pinto A, Santos AS, Figueiredo P, Sarmento A, Santos L. SARS-CoV-2-related multisystem inflammatory
\end{abstract} syndrome in adults. EJCRIM 2021;8: doi:10.12890/2021_003025.

Conflicts of Interests: The authors declare there are no competing interests.

This article is licensed under a Commons Attribution Non-Commercial 4.0 License

\section{ABSTRACT}

Adults infected with SARS-CoV-2 may develop a multisystem inflammatory syndrome (MIS-A) characterized by elevated inflammatory markers and multisystem organ involvement. We report the case of a patient who presented with fever and vomiting at hospital admission. He tested positive for SARS-CoV-2 infection and blood tests showed elevated inflammatory markers. The patient developed acute cardiac dysfunction and shock in less than 24 hours and the echocardiogram revealed an LVEF of $30 \%$. He was discharged 3 weeks later fully recovered. MIS-A should be considered if a compatible syndrome is observed in patients with evidence of SARS-CoV-2 infection by PCR test or serology.

\section{LEARNING POINTS}

- Multisystem inflammatory syndrome should be considered in young adults presenting with shock and elevated inflammatory markers.

- Multisystem inflammatory syndrome may be highly responsive to parenteral steroids.

\section{KEYWORDS}

SARS-CoV-2, multisystem inflammatory syndrome, adult patients

\section{INTRODUCTION}

Coronavirus disease 2019 (COVID-19) is a respiratory infection caused by severe acute respiratory syndrome coronavirus 2 (SARS-CoV-2); the spectrum of clinical manifestations continues to evolve. Children may develop multisystem inflammatory syndrome (MIS-C) that occurs weeks after SARS-CoV-2 infection and is characterized by fever, elevated inflammatory markers, and multisystem organ involvement ${ }^{[1]}$. Although it was thought to be specific to children, a similar syndrome in adults has been described and designated multisystem inflammatory syndrome in adults (MIS-A) ${ }^{[2]}$.

In October 2020, the Centre for Disease Control and Prevention (CDC) proposed the first MIS-A case definition. This definition has since changed and includes patients aged $\geq 21$ years and hospitalized for $\geq 24$ hours who meet the following clinical (I) and laboratory (II) criteria without a more likely alternative diagnosis: I) clinical: fever for $\geq 24$ hours with at least three clinical criteria (including at least one primary criterion) - A) primary: 1) severe cardiac illness or 2) rash and non-purulent conjunctivitis; B) secondary: 1) new-onset neurological signs or symptoms, 2) shock or hypotension not related to medication, 3) abdominal symptoms or 4) thrombocytopenia; II) laboratory: A) a positive SARS-CoV-2 test for current or recent infection and B) elevated levels of at least two inflammatory markers [2]. Here, we present a case of multisystem inflammatory syndrome associated with SARS-CoV-2 infection in an adult that fulfilled the CDC MIS-A case definition. 


\section{CASE DESCRIPTION}

A previously healthy 37-year-old Bissau-Guinean man presented to the emergency department (ED) in Porto, Portugal with fever, odynophagia, enlarged cervical lymph nodes and vomiting for the last 4 days. In the ED he was febrile $\left(39^{\circ} \mathrm{C}\right)$ but remained haemodynamically stable and without signs of respiratory distress. A SARS-CoV-2 polymerase chain reaction (PCR) test was positive. Blood tests showed elevated leukocytes and inflammatory markers (Table 1). Computed tomography (CT) revealed multiple enlarged cervical lymph nodes but no lung or pleural disease (Figs. 1-3). The patient was admitted to the Infectious Diseases department for further investigation.

\begin{tabular}{|c|c|c|c|c|}
\hline Parameter & Units & ED presentation & ICU admission & ICU discharge \\
\hline Haemoglobin & $\mathrm{g} / \mathrm{dl}$ & 13.2 & 11.5 & 13.5 \\
\hline Platelets & $\times 10^{9} \mathrm{cells} / \mathrm{l}$ & 202 & 208 & 307 \\
\hline WBC & $\times 10^{9}$ cells $/ \mathrm{l}$ & 18.94 & 30.53 & 8.03 \\
\hline Neutrophils & $\times 10^{9}$ cells $/ \mathrm{l}$ & 17.99 & 27.78 & 5.17 \\
\hline Lymphocytes & $\times 10^{9}$ cells $/ \mathrm{l}$ & 0.470 & 0.370 & 2.05 \\
\hline Urea & $\mathrm{mg} / \mathrm{dl}$ & 31 & 96 & 36 \\
\hline Creatinine & $\mathrm{mg} / \mathrm{dl}$ & 1.15 & 3.37 & 0.94 \\
\hline Sodium & $\mathrm{mmol} / \mathrm{l}$ & 135 & 142 & 135 \\
\hline Potassium & $\mathrm{mmol} / \mathrm{l}$ & 3.9 & 3.6 & 3.5 \\
\hline Chloride & $\mathrm{mmol} / \mathrm{l}$ & 101 & 104 & 102 \\
\hline Hs-cTnl & $\mathrm{ng} / \mathrm{l}$ & 26.7 & 23009.5 & 17.8 \\
\hline BNP & $\mathrm{pg} / \mathrm{ml}$ & 83.3 & 2153.6 & 14.1 \\
\hline aPTT & $\mathrm{sec}$ & 35.4 & 57.2 & 29.2 \\
\hline INR & & 1.27 & 1.39 & 1.17 \\
\hline CPR & $\mathrm{mg} / \mathrm{l}$ & 293 & 470 & 6.1 \\
\hline Ferritin & $\mathrm{ng} / \mathrm{ml}$ & 890 & 1701 & 238 \\
\hline D-dimers & $\mu \mathrm{g} / \mathrm{ml}$ & 2.97 & 5.86 & 0.5 \\
\hline PCT & $\mathrm{ng} / \mathrm{ml}$ & - & 147.96 & 0.05 \\
\hline
\end{tabular}

Table 1. Laboratory parameters at ED presentation (day 0), ICU admission (day 1) and ICU discharge (day 14).

aPTT, activated partial thromboplastin time; BNP, brain natriuretic peptide; CRP, C-reactive protein; ED, emergency department, Hs-cTnl, high sensitivity cardiac troponin I; ICU, intensive care unit; INR, international normalized ratio; PCT, procalcitonin; WBC, white blood cell count.

The next morning the patient was confused, hypotensive (blood pressure $60 / 30 \mathrm{mmHg}$ ) and anuric. Arterial blood gas analysis showed no respiratory failure and lactate $4 \mathrm{mmol} / \mathrm{l}$. Blood was collected for culture, and the patient started ceftriaxone. Aggressive fluid resuscitation was administered but without haemodynamic response. Septic shock of unknown origin was suspected, and the patient was admitted to the Infectious Diseases intensive care unit (ICU) for vasopressor support.

Serial electrocardiograms (ECG) showed no abnormalities, but the echocardiogram revealed severe left ventricular systolic dysfunction with an ejection fraction (LVEF) of 30\%. The patient's haemodynamic status deteriorated further. Noradrenaline $1.5 \mu \mathrm{g} / \mathrm{kg} / \mathrm{min}$ was administered and he was intubated and started inotropic support (dobutamine $1 \mu \mathrm{g} / \mathrm{kg} / \mathrm{min}$ ). Blood tests showed elevated inflammatory markers, acute myocardial injury, acute kidney injury and coagulopathy (Table 1). Antibiotics were changed to piperacillin-tazobactam plus linezolid and hydrocortisone 50 mg every 6 hours was started. 
European Journal

of Case Reports in

Internal Medicine

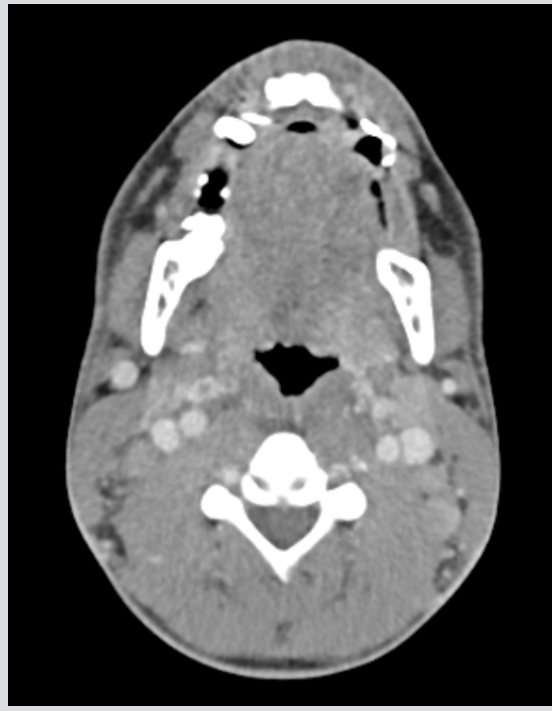

Figure 1. Cervical CT: Left enlarged cervical lymph nodes (axial view)

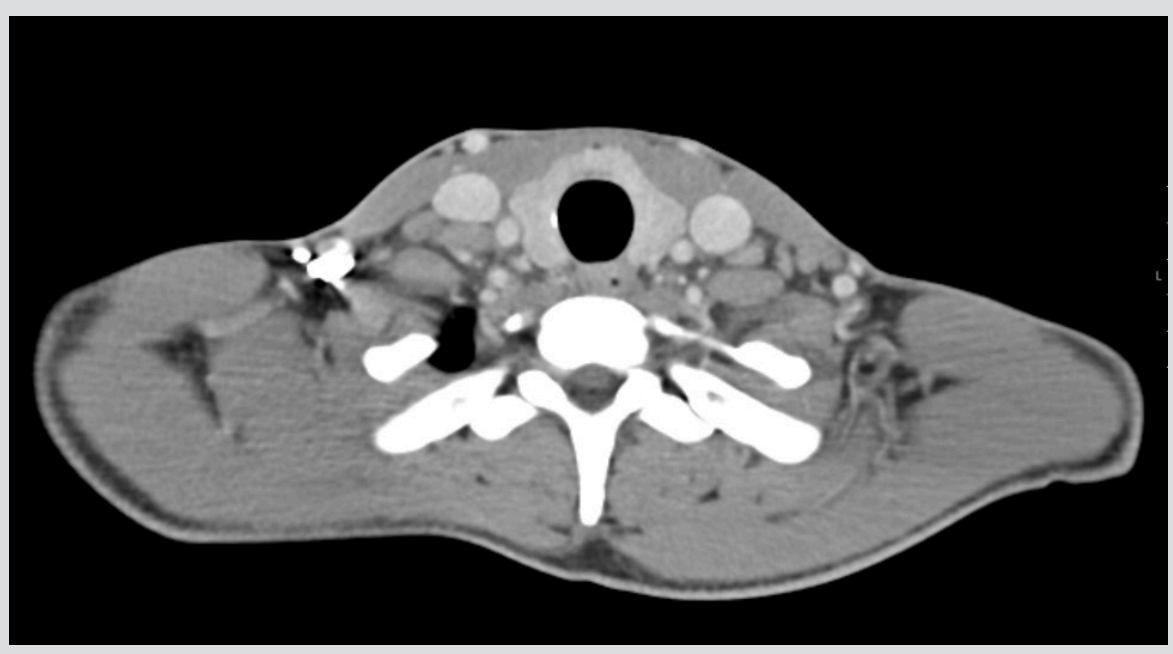

Figure 2. Cervical CT: Right enlarged cervical lymph nodes (axial view)

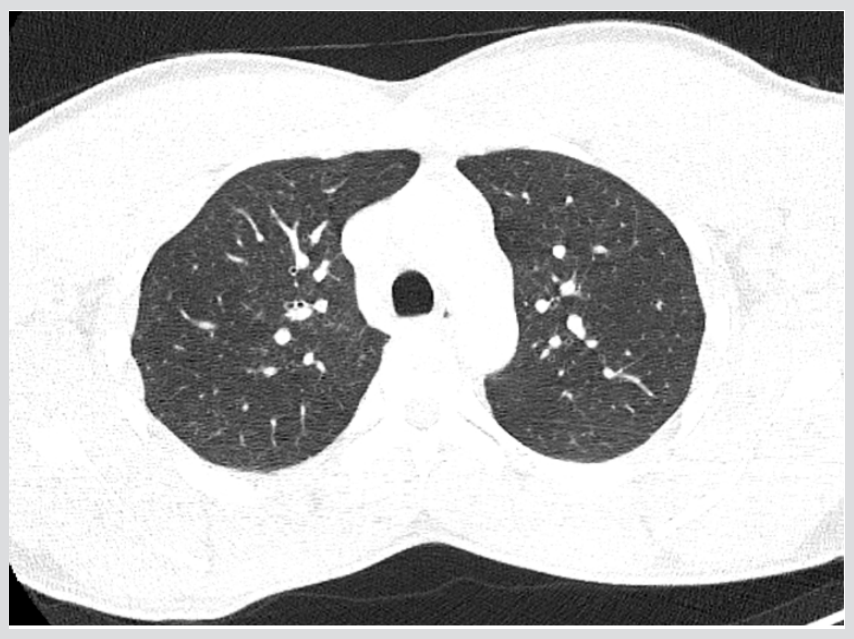

Figure 3. Thoracic CT: No lung or pleural disease was observed (axial view)

The patient's condition improved the next day. Vasopressor/inotropic support was suspended 4 days later and after 1 week the echocardiogram revealed preserved biventricular systolic function with an LVEF of 55\%. Blood, sputum, urine and lymph node cultures were negative and antibiotics were suspended after 7 days. By the end of the second week, the patient had been extubated and discharged from the ICU. Cardiac magnetic resonance (CMR) imaging was performed 1 month later and revealed preserved biventricular systolic function and no oedema or fibrotic or scar tissue.

\section{DISCUSSION}

This case report describes an adult patient with multisystem inflammatory syndrome associated with SARS-CoV-2 infection. In October 2020, the CDC proposed the first MIS-A case definition ${ }^{[3]}$ and a case series review was published in March $2021^{[4]}$.

The pathophysiology of MIS-A is currently unknown. Patients who had typical COVID-19 symptoms before MIS-A diagnosis developed this syndrome 2-5 weeks later. At least one-third had positive serology for SARS-CoV-2 but a negative PCR test ${ }^{[3]}$. These two facts suggest acute cardiac dysfunction and shock may be caused by SARS-CoV-2-triggered systemic inflammation rather than the cytopathic effects of the virus itself. 
MIS-A patients frequently presented with fever (80.4\%), gastrointestinal symptoms including vomiting, diarrhoea and abdominal pain (72.5\%), and respiratory symptoms including cough and dyspnoea (54.9\%) ${ }^{[4]}$. Kawasaki-like features, such as bilateral conjunctivitis, oral mucosal changes or diffuse rash, were less frequently described. Leukocytes, CPR, ferritin and D-dimers were the most common elevated inflammatory markers.

Up to $80 \%$ of the patients had evidence of cardiac injury including ECG abnormalities, elevated troponin levels and/or an echocardiogram with systolic dysfunction, and more than half needed vasopressors ${ }^{[4]}$. Patients who underwent CMR had inconsistent findings, varying from non-specific late gadolinium enhancement to diffuse oedema compatible with a diagnosis of myocarditis according to the Lake Louise criteria ${ }^{[5,6]}$.

In the case presented, the patient had no previous history of COVID-19 and tested positive on a SARS-CoV-2 nasopharyngeal PCR test, which suggests a case of MIS-A presenting early. The patient developed acute cardiac dysfunction and shock less than 24 hours after admission, which shows the potential fulminant course and severity of this condition. Here, the most plausible explanation for the acute cardiac dysfunction is myocarditis. Unfortunately, we only had access to CMR 1 month after admission and could not corroborate this hypothesis. After multidisciplinary evaluation, we chose not to perform an endomyocardial biopsy given the rapid and favourable evolution in the ICU.

Differential diagnoses include severe COVID-19, bacterial sepsis, viral myocarditis, stress cardiomyopathy and coronary artery disease, and these should be excluded if possible. Treatment guidelines for MIS-C patients recommend a stepwise combination of intravenous immunoglobulin (IVIG) and steroids ${ }^{[7]}$. However, the best treatment approach for MIS-A patients has not yet been defined. Up to $20 \%$ of patients were admitted to ICU and most were treated with steroids $(60.8 \%)$ and IVIG $(37.3 \%)^{[4]}$. The majority survived and were discharged from the hospital within 3 weeks.

\section{CONCLUSION}

This case report describes an early presenting and severe case of MIS-A associated with SARS-CoV-2 infection in a previously healthy patient. Clinicians should be aware of MIS-A because early recognition and intensive care may determine the prognosis of these patients. However, further research is needed to elucidate the pathogenesis, clinical manifestations and optimal treatment.

\section{REFERENCES}

1. Centers for Disease Control and Prevention. Information for healthcare providers about multisystem inflammatory syndrome in children (MIS-C). Available from: https:// www.cdc.gov/mis-c/hcp/ (accessed 10 Oct 2021).

2. Centers for Disease Control and Prevention. Multisystem inflammatory syndrome in adults (MIS-A). Available from: https://www.cdc.gov/mis-c/mis-a.html (accessed 10 Oct 2021).

3. Morris SB, Schwartz NG, Patel P, Abbo L, Beauchamps L, Balan S, et al. Case series of multisystem inflammatory syndrome in adults associated with SARS-CoV-2 infection United Kingdom and United States, March-August 2020. MMWR Morb Mortal Wkly Rep 2020;69(40):1450-1456. doi:10.15585/mmwr.mm6940e1.

4. Bastug A, Aslaner H, Bilir YA, Kemirtlek N, Gursoy FM, Bastug S, et al. Multiple system inflammatory syndrome associated with SARS-CoV-2 infection in an adult and an adolescent. Rheumatol Int 2021;41:993-1008. doi:10.1007/s00296-021-04843-1.

5. Chau VQ, Giustino G, Mahmood K, Oliveros E, Neibart E, Oloomi M, et al. Cardiogenic shock and hyperinflammatory syndrome in young males with COVID-19. Circ Heart Fail 2020;13(10):e007485. doi:10.1161/CIRCHEARTFAILURE.120.007485.

6. Hékimian G, Kerneis M, Zeitouni M, Cohen-Aubart F, Chommeloux J, Bréchot N, et al. COVID-19 acute myocarditis and multisystem inflammatory syndrome in adult intensive and cardiac care units. Chest 2020;159(2):657-662. doi:10.1016/j.chest.2020.08.2099.

7. Henderson LA, Canna SW, Friedman KG, Gorelik M, Lapidus SK, Bassiri H, et al. American College of Rheumatology clinical guidance for multisystem inflammatory syndrome in children associated with SARS-CoV-2 and hyperinflammation in pediatric COVID-19: version 2. Arthritis Rheumatol 2021;73(4):e13-29. doi:10.1002/art.41616. 